marks key developmental genes in embryonic stem cells. Cell. 2006;125(2):315-326.

21. Vastenhouw NL, Schier AF. Bivalent histone modifications in early embryogenesis. Curr Opin Cell Biol. 2012;24(3):374-386

22. Pan G, et al. Whole-genome analysis of histone $\mathrm{H} 3$ lysine 4 and lysine 27 methylation in human embryonic stem cells. Cell Stem Cell. 2007;1(3):299-312.

23. Guenther MG, Levine SS, Boyer LA, Jaenisch R, Young RA. A chromatin landmark and transcription initiation at most promoters in human cells. Cell. 2007;130(1):77-88.

24. Shi X, et al. ING2 PHD domain links histone H3 lysine 4 methylation to active gene repression. Nature. 2006;442(7098):96-99.

25. Greer EL, Shi Y. Histone methylation: a dynamic mark in health, disease and inheritance. Nat Rev Genet. 2012;13(5):343-357.

26. Levine M. Paused RNA polymerase II as a developmental checkpoint. Cell. 2011;145(4):502-511.

27. Katsuta H, et al. Subpopulations of GFP-marked mouse pancreatic $\beta$-cells differ in size, granularity, and insulin secretion. Endocrinology. 2012; 153(11):5180-5187.

28. Truax AD, Greer SF. ChIP and Re-ChIP assays: investigating interactions between regulatory proteins, histone modifications, and the DNA sequences to which they bind. Methods Mol Biol. 2012;809:175-188

29. Xie R, et al. Dynamic chromatin remodeling mediated by polycomb proteins orchestrates pancreatic differentiation of human embryonic stem cells. Cell Stem Cell. 2013;pii:S1934-5909(12)00706-0.

30 . Lu J, et al. $\alpha$ cell-specific Men 1 ablation triggers the transdifferentiation of glucagon-expressing cells and insulinoma development. Gastroenterology. 2010;138(5):1954-1965.e1958.

31. Agarwal SK, Jothi R. Genome-wide characterization of menin-dependent $\mathrm{H} 3 \mathrm{~K} 4 \mathrm{me} 3$ reveals a specific role for menin in the regulation of genes implicated in MEN1-like tumors. PLoS One. 2012 7(5):e37952.
32. James LI, et al. Discovery of a chemical probe for the L3MBTL3 methyllysine reader domain [published online ahead of print January 6, 2013]. Nat Chem Biol. doi:10.1038/nchembio.1157.

33. Fomina-Yadlin D, et al. Small-molecule inducers of insulin expression in pancreatic-cells. Proc Natl Acad SciU S A. 2010;107(34):15099-15104.

34. Gu W, et al. Long-term inhibition of the glucagon receptor with a monoclonal antibody in mice causes sustained improvement in glycemic control, with reversible alpha-cell hyperplasia and hyperglucagonemia. J Pharmacol Exp Ther. 2009;331(3):871-881.

35. Gelling RW. Lower blood glucose, hyperglucagonemia, and pancreatic $\alpha$ cell hyperplasia in glucagon receptor knockout mice. Proc Natl Acad Sci U S A. 2003;100(3):1438-1443

36. Longuet $\mathrm{C}$, et al. Liver-specific disruption of the murine glucagon receptor produces $\alpha$-cell hyperplasia: evidence for a circulating $\alpha$-cell growth factor [published online ahead of print November 16 2012]. Diabetes. doi:10.2337/db11-1605.

\title{
Virgin birth: engineered heart muscle from parthenogenetic stem cells
}

\author{
Sara J. McSweeney and Michael D. Schneider
}

British Heart Foundation Centre of Research Excellence, National Heart and Lung Institute, Imperial College London, London, United Kingdom.

\begin{abstract}
Cardiac muscle restitution, or true regeneration, is an unmet need in the treatment of myocardial infarction (MI), prompting a decade of study with stem cells of many kinds. Among key obstacles to effective cardiac cell grafting are the cost of autologous stem cell-derived cardiomyocytes, the ethical implications of using embryonic stem cell (ESC) products, immunological barriers to allogeneic cells, functional maturation beyond just the correct lineage decision, and the lack of durable engraftment. In this issue of the JCI, Didié and colleagues show that cardiomyocytes made from parthenogenetic stem cells (PSCs) and deployed as engineered heart muscle (EHM) may overcome all of these formidable barriers.
\end{abstract}

Survival after MI has increased dramatically over the last 30 years, chiefly due to improvements in restoring blood flow to the ischemic heart and in preventing longterm dilation and wall thinning. However, these remedies do not address the underlying cause of biomechanical dysfunction after damage, namely the death of up to 1 billion cardiomyocytes (1). Under normal circumstances, myocyte replacement is measurable but scant (2). Therefore, strategies are being developed to replace the lost cardiomyocytes using various types of stem or progenitor cells. Clinical investigations of bone marrow populations including mesenchymal stem cells have shown

Conflict of interest: The authors have declared that no conflict of interest exists.

Citation for this article: J Clin Invest. doi:10.1172/ JCI67961. encouraging, though limited, benefits and are currently in phase III trials; however, these are now envisioned as working chiefly through angiogenesis and paracrine effects, not myocyte replacement (3). Heart-derived progenitor cells with clearer potential for cardiac muscle creation have recently completed phase I safety trials (4).

Pluripotent cells with the capacity to generate all the cell types of the body are an alternative strategy for heart repair that have long been studied in the laboratory, but have been slow to find their way into the clinic. Injection of pluripotent cells themselves is problematic due to their ability to form teratomas (5), so therapeutic use of these would require the rigorous purification of stem cell-derived cardiomyocytes or perhaps their committed precursors. Translation of such work to the clinic has also been hindered by many other issues regarding the use of human ESCs, including ethical disputes and the fundamental challenge of immunological rejection. For this reason, immunologically privileged approaches to generate heart muscle from pluripotent stem cells must be considered. Induced pluripotent stem cells (iPSCs) are readily created from skin fibroblasts or blood, and do not raise the ethical objections associated with ESCs. Like ESCs they can differentiate into cardiomyocytes, but can, in principle, be generated as a patient-specific therapy (6). However, the epigenetic memory of these cells could bias them toward certain fates (7), their immunological status has been questioned (6), and the logistics of "bespoke" therapy are far more complex than the hypothetical universal donor.

\section{Single-parent stem cells}

As a new option for cell therapy, Didié et al. show in the current study that PSCs could be an alternative to ESCs in cardiac regeneration (8), as they do not have the same ethical implications. Parthenogenesis (Greek for "virgin birth") is a natural form of asexual reproduction observed in plants, invertebrates, fish, amphibians, and reptiles. During the formation of a normal mammalian embryo, oocytes are arrested in metaphase II until fertilization, when 


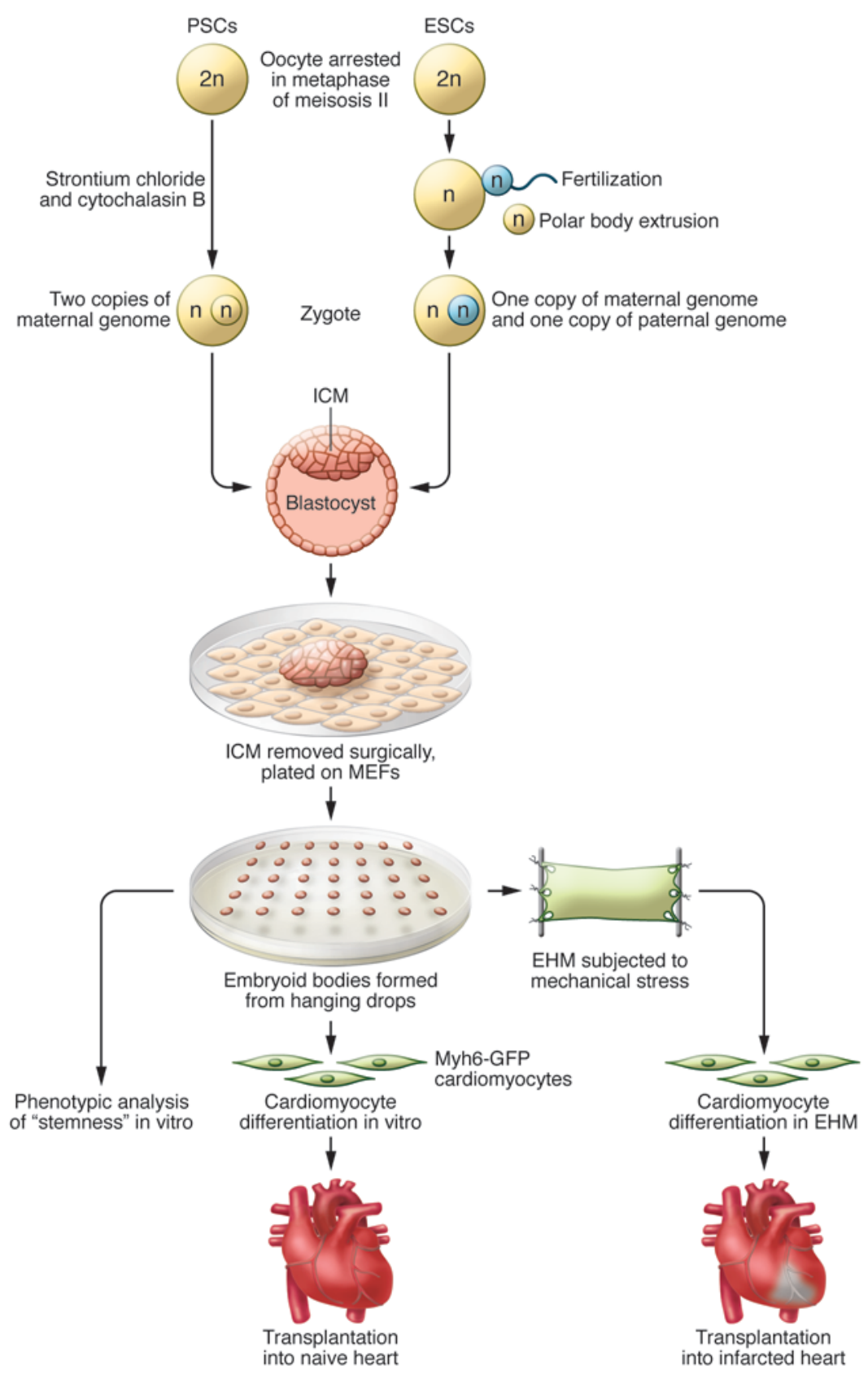

intracellular calcium oscillations enable the arrested oocyte to progress through meiosis. The surplus set of maternal chromosomes is then extruded as a polar body, the zygote forms a blastocyst, and ESC lines may be generated from the inner cell mass (ICM) (9). In contrast, to produce PSCs, intracellular calcium oscillations are provoked artificially with chemicals or electrical impulses mimicking fertilization $(8,9)$. A diploid genome is maintained by treating the cells with cytochalasin B to prevent the extrusion of the second polar body $(8,9)$. The process of making stem cells from a parthenote then closely follows that used for normal diploid embryos, including removal of the ICM, plating on a feeder layer of cells, isolating outgrowths, and growing embryoid bodies in hanging droplets (Figure 1 and ref. 8).

Didié et al. show convincingly that the resulting PSCs have a very similar phenotype to ESCs. They expressed the "stemness" factors Oct3/4, SSEA 1 , and Nanog, demonstrated alkaline phosphatase activity, and had growth kinetics indistinguishable from the well-characterized R1-ESC line. Principal component

\section{Figure 1}

EHM from PSC-derived cardiomyocytes. (Above) PSCs and ESCs are produced from oocytes arrested in meiosis II and stimulated to form a zygote, either by fertilization by sperm (ESC) or artificially with strontium chloride (PSC). In the production of ECSs, a polar body containing one set of the maternal genome is subsequently extruded as a polar body, whereas in PSC production this is prevented by cytochalasin B. Both processes result in 2 copies of the genome, although in the case of PSCs both are from the mother. PSCs, being uniparental in origin, are advantageous for immunological matching. From this stage onward, the production of stem cells from the parthenote follows that from embryos. The ICM of the blastocyst is removed and plated on mouse embryonic fibroblasts (MEFs), outgrowths are isolated, and embryoid bodies are grown in hanging drops. Undifferentiated or differentiated cells are then used for analysis in vitro and in vivo. (Below) Stem cells having made the lineage decision to become cardiomyocytes are induced to undergo functional maturation by a tissue-engineering approach including static mechanical stretch and inclusion of nonmyocytes. The use of EHM also promotes persistent engraftment after MI. analysis of the transcriptome demonstrated that PSCs segregate with ESCs and iPSCs. Three-germ-layer multilineage potential was confirmed, as the cells differentiated into ectoderm, mesoderm, and endoderm, confirming the findings in other studies of mouse, human, and primate PSCs $(9,10)$. Concordant with the presence of genomic aberrations and copy number variation in ESCs, iPSCs, and even adult cardiac progenitors, similar changes were found in PSCs, likely due in part to selective pressures in culture (11, 12). Growing cells in physiological $\mathrm{O}_{2}$ con- 
centrations $(1 \%-7 \%)$ rather than normoxia $\left(20 \% \mathrm{O}_{2}\right)$ may reduce hyperoxic stress and improve the genetic stability of stem cells (12), and should be explored with PSCs in future studies. The relevant level and duration of safety surveillance on this matter remains an unsettled question for longterm processed cells of all kinds.

\section{The immunological match game and cell banking}

An individual's cells express combinations of MHCs, in humans called human leukocyte antigens (HLAs), enabling immune cells to distinguish between self and nonself (13). Mismatch of the transplanted cells' or tissues' MHC genes with those of the recipient results in transplant rejection, which can be prevented by treatment with immunosuppressive drugs; however, such drugs can have devastating side effects (13). From an immunological perspective, cell therapy should ideally use autologous cells, but individualized cell line creation is time consuming, costly, and subject to variation in key properties. Allogenic but HLA-matched cells are an alternative, but maintenance of the required cell banks is inherently expensive, given the number of human MHC alleles (almost 1,500) and the inheritance of more than 12 separate alleles from each parent (14). Conceptually, cells that are homozygous for the key HLA loci could be implanted into homoand heterozygotic recipients, reducing the number of lines required to match most of the clinical population; however, such homozygosity is rare (14). PSCs contain a duplicated haploid genome inherited from just 1 parent and therefore are predominantly homozygous, increasing the chances of being immunocompatible (8). Although rejection can result from the absence of selfalleles, not just the presence of nonself ones (9), in the current study PSC grafts were well tolerated as long as no mismatched allele was present. In silico predictions by the authors suggest that less than 100 haploidentical cell lines are required to match greater than $90 \%$ of the human population, suggesting that a PSC bank is a tractable way of storing cells suitable for the majority of future recipients (8).

\section{Engineering cell maturity}

Didié et al. show that mouse PSCs can differentiate into structurally mature cardiomyocytes with well-developed sarcomeres in accordance with other studies that have demonstrated that the same is true for PSCs from primates and humans $(8,10)$. The PSC-derived cardiomyocytes had the electrophysiological properties of mature cardiomyocytes and were able to engraft into the uninjured myocardium, coupling electrically to the recipient heart, with $\mathrm{Ca}^{2+}$-transient kinetics comparable to those of the host tissue. There have been conflicting reports regarding the arrhythmogenic potential of engrafted cardiomyocytes $(15,16)$, but an encouraging recent study demonstrated that human ESC-derived cardiomyocytes can electrically integrate with the host myocardium and contract synchronously in guinea pigs (17). Because pluripotent cell-derived cardiomyocytes exhibit a mix of ventricular-, atrial-, Purkinje-, and pacemaker-like properties, it is conceivable that purification by sublineage may be advantageous in the future to minimize spontaneous electrical activity in grafted cells.

Biomaterials have been developed to augment the retention and survival of cells in the myocardium, which is low regardless of the method of delivery. Injectable biomaterials such as alginate, fibrin, and polyethylene glycol can be functionalized with growth factors to enhance not only cell retention, but also cell survival, proliferation, and differentiation (18). Cellular and acellular cardiac patches have improved cardiac function and reduced remodeling in experimental models and the MAGNUM trial (18). To aid delivery in the current study, Didié et al. used EHM composed of several layers of PSC-derived cardiomyocytes. As part of the production process, these constructs were subjected to mechanical stretch to aid the maturation of the cardiomyocytes, resulting in EHMs that spontaneously contract and contain cardiomyocytes with sarcomeric cross-striations (19). Previous work from the group used a neonatal rat cardiomyocyte-populated construct implanted onto an infarcted rat heart to improve fractional shortening and prevent left ventricular dilation (19). Analogously, EHMs incorporating PSC-derived cardiomyocytes increased the anterior wall thickness after MI and enhanced wall contractility (8). In addition, Didié et al. demonstrated the presence of capillaries in the grafts, although the origin of this endothelial growth is yet to be determined.

\section{Are PSCs a panacea for cardiac repair?}

In summary, Didié and colleagues have shown highly laudable and compelling evi- dence that mouse PSCs can differentiate into mature, functional cardiomyocytes which in turn can integrate with recipient myocardium and be tolerated as MHCmatched allogenic cells in the absence of immunosuppression. While this is not the first report using PSCs for cardiac myogenesis and repair (5), Didié et al. address issues critical for the eventual translation of this technology to clinical medicine: decreased immunogenicity and augmentation of cardiomyocyte maturation. One unanswered question is how best to promote vascularization of three-dimensional grafts: perhaps seeding EHMs with a second cell type, or using even smarter biomaterials? Immunocompatibility makes PSC-derived cells a preferred candidate for stem cell banking, however, the alternatives should be kept in mind. Engineering ESCs to suppress HLA expression (13) could obviate the most unique present advantage held by PSCs, and adult heart-derived cells might be sufficiently MSC-like to be used as allogeneic products (20). Nevertheless, this multifaceted study by Didié and colleagues clearly and convincingly demonstrates that PSCs are a potent alternative to the other cells proposed to regenerate the heart.

Address correspondence to: Michael D. Schneider, British Heart Foundation Centre of Research Excellence, Imperial College London, Imperial Centre for Translational and Experimental Medicine, Room 336, Du Cane Road, London W12 0NN, United Kingdom. Phone: 011.44.20. 7594.3027; Fax: 011.44.20.7594.3015; E-mail: m.d.schneider@imperial.ac.uk

1. Mercola M, Ruiz-Lozano P, Schneider MD. Cardiac muscle regeneration: lessons from development. Genes Dev. 2011;25(4):299-309.

2. Senyo SE, et al. Mammalian heart renewal by preexisting cardiomyocytes [published online ahead of print December 5, 2012]. Nature. doi:10.1038/ nature11682.

3. Dimmeler S, Zeiher AM, Schneider MD. Unchain my heart: the scientific foundations of cardiac repair. J Clin Invest. 2005;115(3):572-583.

4. Ptaszek LM, Mansour M, Ruskin JN, Chien KR. Towards regenerative therapy for cardiac disease. Lancet. 2012;379(9819):933-942.

5. Liu Y, et al. Transplantation of parthenogenetic embryonic stem cells ameliorates cardiac dysfunction and remodelling after myocardial infarction. Cardiovasc Res. 2013;97(2):208-218.

6. Yamanaka S. Induced pluripotent stem cells: past, present, and future. Cell Stem Cell. 2012; 10(6):678-684

7. Bar-Nur O, Russ HA, Efrat S, Benvenisty N. Epigenetic memory and preferential lineage-specific differentiation in induced pluripotent stem cells derived from human pancreatic islet beta cells. Cell Stem Cell. 2011;9(1):17-23.

8. Didié $M$, et al. Parthenogenetic stem cells for 
tissue-engineered heart repair. J Clin Invest. 2013; 123(3):1285-1298.

9. Kim K, et al. Histocompatible embryonic stem cells by parthenogenesis. Science. 2007;315(5811):482-486.

10. Zimmermann WH. Embryonic and embryonic-like stem cells in heart muscle engineering. J Mol Cell Cardiol. 2011;50(2):320-326.

11. Maitra A, et al. Genomic alterations in cultured human embryonic stem cells. Nat Genet. 2005; 37(10):1099-1103.

12. Li TS, Marban E. Physiological levels of reactive oxygen species are required to maintain genomic stabil- ity in stem cells. Stem Cells. 2010;28(7):1178-1185.

13. Figueiredo C, Horn PA, Blasczyk R, Seltsam A. Regulating MHC expression for cellular therapeutics. Transfusion. 2007;47(1):18-27.

14. Rao MS, Auerbach JM. Estimating human embryonic stem-cell numbers. Lancet. 2006;367(9511):650.

15. Liao SY, et al. Proarrhythmic risk of embryonic stem cell-derived cardiomyocyte transplantation in infarcted myocardium. Heart Rhythm. 2010;7(12):1852-1859.

16. Roell W, et al. Engraftment of connexin 43-expressing cells prevents post-infarct arrhythmia. Nature. 2007;450(7171):819-824
17. Shiba Y, et al. Human ES-cell-derived cardiomyocytes electrically couple and suppress arrhythmias in injured hearts. Nature. 2012;489(7415):322-325.

18. Segers VF, Lee RT. Biomaterials to enhance stem cell function in the heart. Circ Res. 2011;109(8):910-922.

19. Zimmermann WH, et al. Engineered heart tissue grafts improve systolic and diastolic function in infarcted rat hearts. Nat Med. 2006;12(4):452-458.

20. Le Blanc K, et al. Mesenchymal stem cells for treatment of steroid-resistant, severe, acute graftversus-host disease: a phase II study. Lancet. 2008; 371(9624):1579-1586

\section{Bathing the brain}

Warren J. Strittmatter

Departments of Medicine (Neurology) and Neurobiology, Duke University Medical Center, Durham, North Carolina, USA.

\begin{abstract}
The brain and spinal cord are surrounded by cerebrospinal fluid, which provides a mechanically stable environment for these delicate structures against the forces of gravity and sudden acceleration and deceleration. Neurons and glia comprising the parenchyma of the brain are enveloped in their microenvironment by interstitial fluid. Interstitial fluid has long been considered to be unaffected by the production and flow of cerebrospinal fluid outside the brain parenchyma. However, two recent papers by Iliff et al. demonstrate that cerebrospinal fluid enters the deep substance of the brain, mixes with the interstitial fluid surrounding neurons and glia, and plays an important role in the exchange and clearance of molecules in the interstitial space of the central nervous system.
\end{abstract}

Hippocrates observed that the brain is suspended in a fluid, but the functions of that cerebrospinal fluid (CSF) vital to brain function have only been slowly revealed. Francois Magendie compared the brain suspended in CSF to the fetus suspended in amniotic fluid, and indeed, CSF does provide mechanical support for the brain. Since the brain is neutrally buoyant in this environment, CSF provides protection of neural tissue from the force of gravity and from sudden changes in velocity during head impact. The production of CSF by the choroid plexus in the lateral ventricles, its transit through the third and fourth ventricles, its coursing over the exterior surface of the brain and spinal cord, and its ultimate uptake at the arachnoid granulations in the large draining veins was not fully described until the early 1900s (Figure 1A and ref. 1).

\section{Into the soup}

Virtually every cell throughout the body is surrounded by interstitial fluid, which

Conflict of interest: The author has declared that no conflict of interest exists.

Citation for this article: J Clin Invest. doi:10.1172/ JCI68241. plays critical roles in cellular homeostasis, including maintaining osmotic pressure, supplying nutrients, and equilibrating the concentrations of molecules by diffusion. In tissues throughout the body, interstitial fluid is slowly exchanged by intermixing with lymph that transits unidirectionally through the tissue to draining lymphatic vessels, first identified anatomically in the 1600s. The convectional flow of lymph mixes with interstitial fluid and facilitates the transit of molecules and cells within the tissue. Brain, however, does not contain such discrete lymphatic vasculature. The hypothesis that brain possesses a "lymphatic" system with the same physiologic functions, but with different anatomy than that found throughout the remainder of the body, was initially proposed following the observations that CSF is rapidly removed from the neonatal brain, even though the venous system in the neonate contains only few arachnoid granulations, and, additionally, that CSF flows abundantly and unidirectionally along the cribriform plate in the nose, which contains no arachnoid granulations. Injection of radiolabeled albumin into the CSF revealed that half of the albumin tracer eventually drains from the cribriform plate into the cervical lymphatic system $(2,3)$. CSF also drains into the lymphatic system through the sheaths of cranial and spinal nerves (4).

\section{Mapping the flow}

Recently, Iliff et al. demonstrated that the directional flow of CSF through the parenchyma of the brain mixes with brain interstitial fluid and facilitates its clearance (5). In this issue of JCI, this group used nuclear MRI to visualize and quantify the pathway in the intact organism, a critical advance in translating this knowledge to the clinic (6).

Iliff et al. injected a fluorescent tracer into the CSF at different sites and initially observed that tracer injected into the lateral ventricles, the site of CSF production, minimally diffused into the parenchyma of the brain, while in contrast, tracer injected into the cisternal space surrounding the external surface of the brain entered the brain parenchyma. Two-photon laser scanning microscopy revealed that fluorescent dye injected into the cisternal space entered the deep brain parenchyma by traveling along the outside of cerebral arteries, entering the brain via the para-arterial channels of the Virchow-Robin space (6). Once deep into the brain, dye diffused into the parenchyma, bathing neurons and glia and mixing with the interstitial fluid. Within the parenchyma, CSF mixed with interstitial fluid, subsequently traveled along the external surface of large caliber veins, and reentered either the subarachnoid space or drained into the cervical lymphatic system (Figure 1B). This demonstration that CSF robustly transits through the parenchyma of the brain and mixes with interstitial fluid has important implications for 